\title{
Reversing sex role reversal: compete only when you must
}

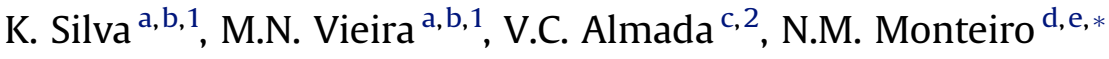 \\ ${ }^{\text {a }}$ Faculdade de Ciências da Universidade do Porto \\ ${ }^{\mathrm{b}}$ CIIMAR (Centro Interdisciplinar de Investigação Marinha e Ambiental) \\ ${ }^{\mathrm{C}}$ Instituto Superior de Psicologia Aplicada \\ ${ }^{\mathrm{d}}$ CEBIMED (Centro de Estudos Biomédicos), Faculdade de Ciências da Saúde da Universidade Fernando Pessoa \\ ${ }^{\mathrm{e}} \mathrm{CIBIO}$ (Research Center In Biodiversity and Genetic Resources)
}

\section{A R T I C L E I N F O}

Article history:

Received 2 July 2009

Initial acceptance 17 September 2009

Final acceptance 29 December 2009

Available online 25 January 2010

MS. number: 09-00445R

\section{Keywords:}

black striped pipefish

competition

female-female-interaction

mating tactics

operational sex ratio (OSR)

sex role reversal

Syngnathidae

Syngnathus abaster
The operational sex ratio (OSR) is thought to be a major factor influencing the intensity of mating competition and sexual selection. Even though many studies on species with conventional sex roles have shown that alterations in the OSR can either intensify male-male competition or promote female-female competitive interactions, sometimes resulting in a reversal of sex roles, it is not known how, and how quickly, individuals with reversed sex roles respond to fluctuations in this ratio. We tested for a direct influence of adult sex ratios (as a direct estimation of the OSR) on the reproductive behaviour of the sex role-reversed black striped pipefish, Syngnathus abaster. Although imbalances in the OSR effectively modulated the expression of sex roles, with males and females varying in the degree of choosiness and competitive displays, alterations in the sex ratios did not promote a similar response pattern in both sexes. A surplus of males resulted in a reversion to conventional sex roles observed when both sexes coexist in similar numbers, with males competing intensely and exhibiting a conspicuous ornament towards other males. An excess of females, in contrast, did not result in an overall increase in female competitive interactions. Only small, less attractive, females were more prone to compete as the proportion of males decreased. Large females, however, seemed to rely on their greater mating prospects, thereby avoiding the hypothetical costs of intrasexual competition.

(C) 2010 The Association for the Study of Animal Behaviour. Published by Elsevier Ltd. All rights reserved.
The operational sex ratio (OSR: ratio of sexually active males to females producing fertilizable eggs at a given time and place) is thought to be an important determinant of animal sex roles (Emlen \& Oring 1977). By definition, biases in this ratio lead to stronger intrasexual competition for mates in the sex occurring in 'excess' while favouring the evolution of greater selectivity in the choice of mating partners by the sex in 'shortage' (Clutton-Brock \& Parker 1992; Kokko \& Monaghan 2001; Clutton-Brock 2007).

Although sex roles can be typically dichotomized into 'conventional', when males compete more intensely for the access to mating partners, and 'reversed', when female competition is stronger (sensu Vincent et al. 1992), studies have demonstrated that fluctuations in ecological and physical factors influencing reproductive rates can affect the OSR. As a result, the intensity of mating competition (e.g. spider mite, Tetranychus urticae: Enders

\footnotetext{
* Correspondence: N. M. Monteiro, CIBIO, Campus Agrário de Vairão, R. Padre Armando Quintas, 4485-661 Vairão, Portugal.

E-mail address: nmonteir@fc.up.pt (N.M. Monteiro).

K. Silva and M. N. Vieira are at the Departamento de Zoologia e Antropologia, Faculdade de Ciências da Universidade do Porto, Rua do Campo Alegre, 4169-007, Porto, Portugal.

2 V. C. Almada is at ISPA, rua Jardim do Tabaco, 34, 1149-041 Lisboa, Portugal.
}

1993; field cricket, Gryllus pennsylvanicus: Souroukis \& Cade 1993; Japanese medaka, Oryzias latipes: Grant 1995; sand goby, Pomatoschistus minutus: Kvarnemo et al. 1995) or sex roles (Almada et al. 1995) can change. Gwynne \& Simmons (1990) showed, in katydids, that when high-quality food is abundant, males produce spermatophores more rapidly than females produce eggs, thus biasing the OSR towards males and leading to male-male competition. In contrast, when food is scarce, males take longer to produce spermatophores, the OSR is female biased, and females become the more competitive sex (Gwynne \& Simmons 1990). More recently, Forsgren et al. (2004) highlighted a similar pattern in the twospotted goby, Gobiuscus flavescens, by showing that a change in the OSR during the breeding season is accompanied by a marked change in mating competition, with male-male competition and intensive male courtship behaviour being superseded by femalefemale competition and actively courting females. Given the potential for flexibility in vertebrate sex roles, as stressed by Forsgren et al. (2004), it seems imperative to explore the range of variation in selection regimes, namely by determining how, and how quickly, animals respond to changes in the OSR. For instance, we can predict that a sex role-reversed species, where females are the predominant competitors for mates, might show a mirror-like response pattern to variation in the OSR. A surplus of 'ready to 
mate' males would promote male-male competition, reversing the system back into conventional sex roles, while an excess of receptive females would increase female-female competition, thus intensifying sex role reversal.

The family Syngnathidae (pipefishes, seahorses and seadragons), in which either males or females may be subject to greater sexual selection (Vincent et al. 1992; Monteiro et al. 2002; Berglund \& Rosenqvist 2003; Silva et al. 2006), provides a unique opportunity to test the influence of OSR on sex role dynamics. Such a topic, however, remains to be explored fully as the majority of studies have focused on a single species, the broad-nosed pipefish Syngnathus typhle (Berglund 1994, 2005; Vincent et al. 1994; Jones et al. 2000, 2005). In this species, even though the sexes do not seem to differ in several life history parameters (e.g. size, mortality, dispersal) observed allocation strategies have proved to be related not only to sex but also to size (Berglund et al. 2006). While males spent more time foraging, females were prone to reproduce. Smaller individuals, on the other hand, seemed to postpone reproduction and thus increase in size faster (Berglund et al. 2006). Furthermore, the degree of choosiness seemed also to be correlated with the displayed allocation tactics, since individuals committed to mating activities seemed also to be more critical in their choices. These results highlight the importance of considering other life history variables, besides the sex of the individual, in studies aimed at understanding the underlying dynamics of a species' mating system.

We tested for the direct influence of adult sex ratios (as a direct estimation of the OSR) on the mating behaviour of the sex rolereversed (Silva et al. 2006) pipefish, Syngnathus abaster. We addressed four major questions. (1) Can sex ratios per se directly affect courtship and mating competition in S. abaster? (2) Do alterations in the OSR influence males and females alike? (3) Given that, in $S$. abaster, body size is strongly implicated in mate choice (Silva et al. 2007), do larger and more attractive individuals react differently from smaller competitors when the OSR changes? (4) Is there a change in the degree of choosiness when OSR is altered?

\section{METHODS}

\section{Study Animal and Procedure}

Syngnathus abaster is a small brown-green pipefish, with dark or pale spots on the trunk and tail. It has a restricted distribution that includes the Mediterranean, the Black Sea and the Atlantic coast of southwest Europe up to southern Biscay (Dawson 1986). This euryhaline pipefish occurs either in coastal areas or in brackish and fresh waters (Cakic et al. 2002), and can be found mainly among sand, mud or eelgrass meadows, at depths between 0.5 and $5 \mathrm{~m}$, within a temperature range of $8-24^{\circ} \mathrm{C}$. Males have a brood pouch located ventrally on the tail, which consists of two skin folds that come into contact medially with their free edges, where they brood a variable number of eggs that may range from only a few up to 64 (Silva et al. 2006). Females attain larger sizes and have a more pronounced lateral coloration on the trunk. This ornamentation can be amplified, in a similar manner as that described for $S$. typhle (Berglund \& Rosenqvist 2003).

Experimental fish were collected with a hand net, in a salt pond reservoir, at the Ria de Aveiro estuarine lagoon $\left(40^{\circ} 45^{\prime} \mathrm{N}, 8^{\circ} 40^{\prime} \mathrm{W}\right)$, in Portugal. Fish were transported to the laboratory in isothermal containers, where they were maintained in 250-litre aquaria, illuminated by natural light supplemented with $18 \mathrm{~W}$ fluorescent lamps. Tank substrata consisted mainly of sand and plastic seagrass laid to mimic the original habitat. The continuously running sea water was physically and biologically filtered and its temperature was kept constant at $18-19{ }^{\circ} \mathrm{C}$ to allow for continuous breeding (see
Silva et al. 2007). Fish were fed daily with fresh Artemia franciscana nauplii. To maximize the chance of observing matings, mature males (nonpregnant) and females were kept in separate tanks for 3 weeks before the experiment.

Individuals were randomly selected according to sex and size and assigned to 40-litre aquaria where three different sex ratio treatments were constructed: even $\left(49: 4{ }^{\star}\right)$, male biased $\left(29: 60^{\star}\right)$ and female biased $\left(6 \%: 2{ }^{\star}\right)$. Observations in the natural populations from where the fish were captured (2003-2009) showed average monthly sex ratios (number of males/(number of males + number of females)) ranging from 0.11 to 0.65 . Thus, we feel that the results extracted from the selected treatments reflect a real adaptive flexibility rather than a response to an artificially skewed sex ratio. Fish were left to acclimatize for 1 day before the observation period. In all aquaria, an equal number of large and small individuals were used. Size cutoffs for 'large' and 'small' individuals were defined according to Silva et al. (2007), as half a standard deviation below and above the mean size (total length) for each sex (ㅇ: mean $\pm \mathrm{SD}=9.4 \pm 1.38 \mathrm{~cm}$; $\delta$ : mean $\pm \mathrm{SD}=8.5 \pm 1.26 \mathrm{~cm}$; values obtained from prior measurements in the same population). Large males and females were longer than $9.1 \mathrm{~cm}$ and $10.1 \mathrm{~cm}$, respectively. Small males and females were shorter than $7.9 \mathrm{~cm}$ and $8.7 \mathrm{~cm}$ respectively. Ten replicates of each sex ratio treatment were conducted.

Owing to the large number of individuals required to complete all three treatments (120 males and 120 females), and given our inability to capture additional pipefish, some fish were used twice ( 35 nonpregnant males and 21 females) but care was taken to allow for a 'resting period' of at least 1 month. For females, this period allows for the production of new eggs ready to be spawned (K. Silva, unpublished data). For males, we reused only nonpregnant males even though aquarium-kept $S$. abaster males were observed receiving new egg clutches only $1 \mathrm{~h}$ after the release of juveniles. Nearly $65 \%$ of the reused males received eggs. A similar proportion of mating success was observed for the nonreused males (ca. 62\%) thus indicating that reuse of individuals might not have affected reproductive patterns.

Each male and female, recognizable by size (large or small), coloration and specific pigmentation patterns on the head, were observed continuously during 10 min periods over 5 consecutive days (50 min overall observation per fish) and the frequency of each selected behaviour (approach, parallel swimming, flickering and lateral display) directed either to a male or a female was recorded (Table 1). Each mating event was also recorded along with the size class of the individuals involved. Additionally, we recorded the number of successful disruptions of ongoing courtship rituals (interpreted as a form of intrasexual competition, according to Silva et al. 2006; Table 1).

The extension of the observation period over a few days seemed particularly important as preliminary observations suggested that females need a few days to deposit their entire egg clutch.

Table 1

Recorded behaviours and corresponding descriptions (according to Silva et al. 2006)

\begin{tabular}{ll}
\hline Behaviour & Description \\
\hline $\begin{array}{l}\text { Approach } \\
\text { Parallel } \\
\text { swimming }\end{array}$ & $\begin{array}{l}\text { Moving towards another fish } \\
\text { Two fish swimming through the aquarium in a more or less } \\
\text { parallel position }\end{array}$ \\
Flickering & $\begin{array}{l}\text { Rapid and vigorous bends along the main axis of the body } \\
\text { displayed towards another fish } \\
\text { Lateral display }\end{array}$ \\
$\begin{array}{l}\text { Temporary colour ornament displayed in a motionless and } \\
\text { parallel position towards another fish } \\
\text { disruption }\end{array}$ & $\begin{array}{l}\text { When an individual interacts with a courting pair, mainly by } \\
\text { placing its body between the individuals, causing the pair's } \\
\text { ongoing behaviour to stop }\end{array}$ \\
\hline
\end{tabular}


Moreover, males could be expected to be more choosy in the female-biased treatment and thus require more time to copulate, as has been shown in another sex role-reversed pipefish (see Berglund 1994). Also, the selected extent of the observation period would maximize the probability of observing all the selected behaviours, simultaneously diluting any possible dependence in their expression (e.g. if a particular behaviour tends to occur first and/or is more frequent, a short observation period might not allow for the detection of other less frequent or late occurring behaviours). Notwithstanding, we opted to avoid very prolonged observation periods to prevent alterations in the OSR, since a fully occupied male would not be receptive to new egg batches.

Given the different numbers of males and females present in the tested sex ratios, the statistical analysis of the observed inter- and intrasexual interactions (in the form of the four selected behaviours) could lead to misinterpretations. As such, observed frequencies per individual were corrected for the different probabilities of encountering an individual of the same or different sex. For example, the 782 male-male approaches observed in the malebiased treatment over the entire observation period were divided by the proportion of males that could have been encountered (5/7). In respect to courtship disruptions, observed frequencies were corrected for the number of courting pairs that a particular individual could have disrupted in each sex ratio treatment. For example, a male in the even treatment, had, at each moment, the opportunity to disrupt three pairs.

To avoid nonindependence, statistical analysis only included data from one type of interaction per fish (male to male $(M \rightarrow M)$, female to female $(F \rightarrow F)$, male to female $(M \rightarrow F)$ or female to male $(\mathrm{F} \rightarrow \mathrm{M})$ ). For instance, in the 10 male-biased sex ratio replicates, with a total of 30 large and 30 small males, the analysis only included 15 randomly selected observations from large males interacting with males (in the form of all four behaviours) and 15 observations from large males interacting with females (in the form of all four behaviours).

\section{Cluster Analysis}

The mean frequencies of all selected behaviours for both types of interactions (intra- and intersexual) were used to create a similarity matrix (fourth-root transformation, similarity measure: Euclidian distance). A cluster analysis, using the computer software package PRIMER 5 (Plymouth routines in multivariate ecological research Primer-E Ltd, Ivybridge, U.K.), was then conducted (cluster mode: group average) to observe a possible relationship between the tested sex ratios and the types of interactions performed by each sex. If some of the tested sex ratios intensified or depressed the number of interactions, visible clusters would be formed helping us to understand the underlying dynamics of the observed process. As an example, we predicted that female-female interactions would reach the highest values in the female-biased sex ratio. We also predicted that male-male interactions, in the male-biased sex ratio, would also attain high values, thus forming a cluster. Furthermore, this approach would allow for a 'global ordination' of the intra- and intersexual interactions, for all tested sex ratios (e.g. in the femalebiased sex ratio, will females interact more with males or females? And how do these values relate to male-performed interactions in the male-biased sex ratio?).

\section{MANOVAs}

Four multiple analyses of variance (MANOVAs), one for each behaviour (approach, parallel swimming, flickering and lateral display), were conducted to test for possible effects of sex ratio, sex and body size on the frequencies of intra- and intersexual interactions. To obtain homogeneity of variances, data were log transformed in the case of parallel swimming and flickering (intrasexual interactions) and flickering (intersexual interactions). Lateral display data (intersexual interactions) were square-root transformed. Even though the homogeneity of variances assumption was not met for approach and lateral display, solely for intrasexual interactions, we decided to continue with the analysis given the robustness of the $F$ statistic (Lindman 1974).

\section{ACTUS and Chi-square Analysis}

Successful disruptions caused by males or females were analysed with ACTUS (analysis of contingency tables using simulation; Estabrook \& Estabrook 1989). For each sex, a potential association of body size and sex ratio treatments was assessed. Simultaneously, using an observed versus expected chi-square analysis (with Bonferroni adjustments), we assessed the differences between disruptions among large and small individuals within each sex ratio treatment.

A possible association between sex ratios and mating preferences was then assessed with ACTUS. The observed number of sizematched and nonsize-matched pairs was scored for each treatment. Additionally, within the observed nonsize-matched mating pairs formed during the experiment (large males with small females or small males with large females), a possible association between the sex ratio treatment and the size combination between males and females was investigated.

\section{ANOVA}

An orthogonal ANOVA was conducted to determine whether sex ratio and male size affected pregnancy latency period (here interpreted as an indirect measurement of male choosiness, as observed by Berglund 1994).

Post hoc comparisons for ANOVA and MANOVA were conducted using a Newman-Keuls test. All probabilities are two tailed and a significance level of 0.05 was used. Unless otherwise stated, statistical analyses were performed using Statistica 6.1 (Statsoft, Tulsa, OK, U.S.A.).

\section{Ethical Note}

Animals were collected with permission of the Portuguese 'Capitania do Porto de Aveiro'. Most of the fish that enter the salt pond reservoirs eventually die because of water evaporation or water usage for salt production, although some may eventually escape back into the estuary. Nevertheless, since this species migrates from the estuary by September (it cannot be located in the area except during the breeding season; N. M. Monteiro, personal observation), we opted not to release the fish either in the salt pond reservoirs or in the estuary. At the end of the experimental period, all the fish were maintained in the laboratory and used in further studies involving reproduction and larval development.

\section{RESULTS}

\section{Cluster Analysis}

The dendrogram resulting from the cluster analysis identified two major clusters, A and B (Fig. 1). Cluster A shows that intrasexual interactions were greatly reduced when sex ratios were skewed towards a large number of available mating partners (IFM and IMF). Given the sex role reversal observed in this species, male-to-male interactions were also clustered in this group which is basically characterized by the lowest observed frequencies of all the selected 


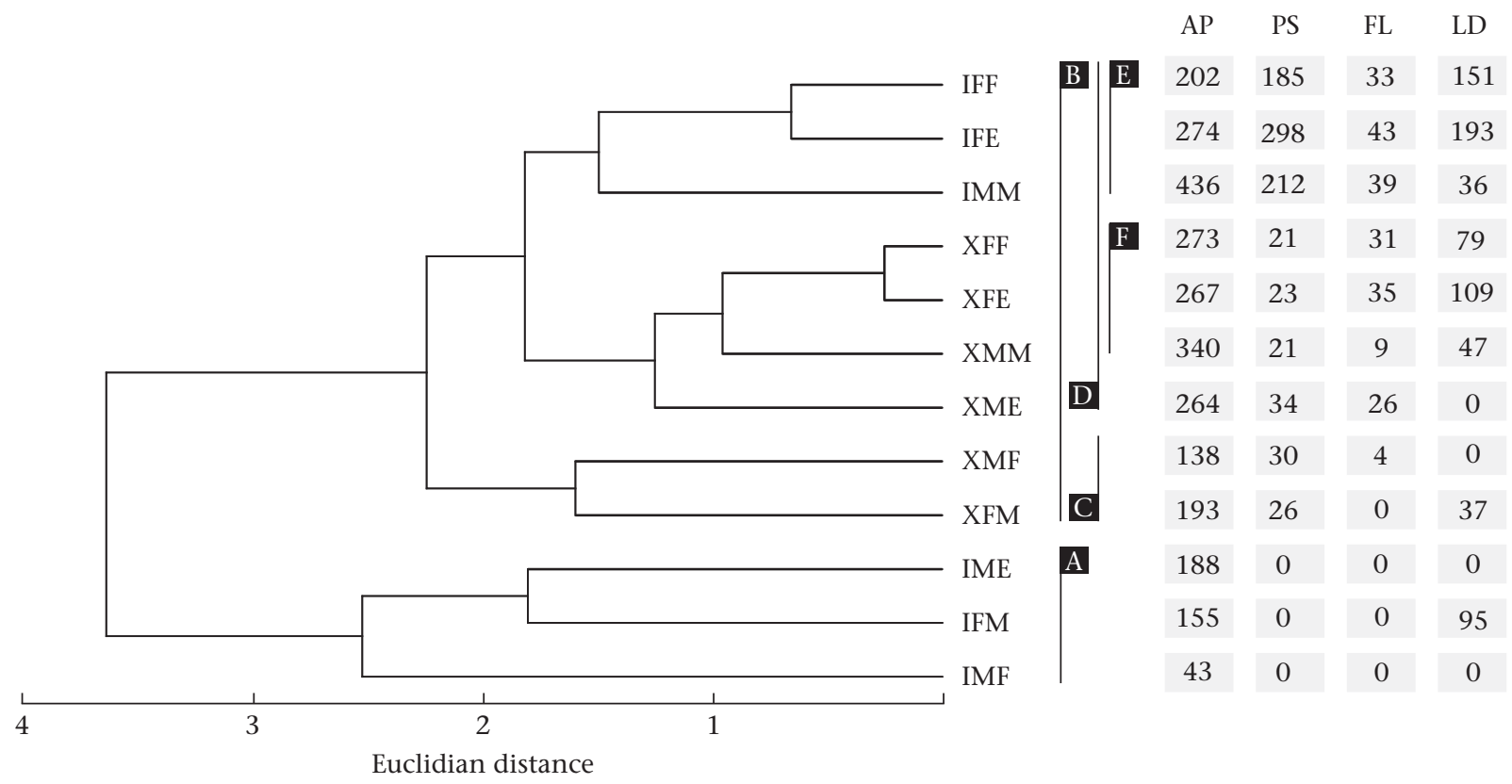

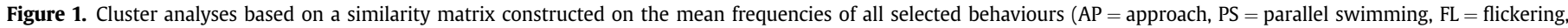

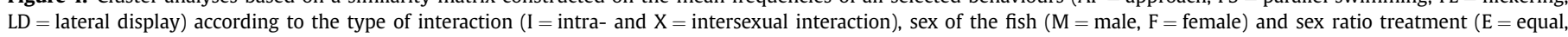
$\mathrm{M}=$ male biased, $\mathrm{F}=$ female biased). For further details see the Results.

behaviours. Cluster B was subdivided into group $C$ which combined intersexual interactions of both males and females when many available partners were present (XMF and XFM), and group D. Within group $\mathrm{D}$, the highest frequencies were recorded for malemale and female-female interactions when more potential competitors existed (IFF and IMM). Again, taking the sex role reversion of $S$. abaster into consideration, under an equal sex ratio female-female competition was also high (IFE). Intermediate values were grouped in cluster F. Even though the selected behaviours are usually described as part of the courtship ritual, we note that the highest frequencies of these behaviours were expressed towards the same sex, when sex ratio treatments were skewed towards more potential competitors.

\section{MANOVAs}

All four MANOVAs showed a significant interaction between the tested sex ratios, sex and focal fish size (Table 2). To identify the contribution of the dependent variables to the significant overall effect, we proceeded to analyse the univariate results (Table 3).

\section{Intrasexual interactions}

In the equal sex ratio treatment (E), female-female interactions were more common than male-male interactions (Fig. 2). While females displayed all four behaviours towards other females, males were only seen approaching other males. Large females interacted more with other females than small ones while no differences were observed between large and small males. Biased sex ratios induced different response patterns in males and females. Male-biased sex ratios induced an increase in the number of male-male interactions, compared to the equal sex ratio treatment, with males displaying all selected behaviours. Also, large and small males differed significantly in activity levels. Large individuals were more interactive than small ones (except for lateral display where no differences were observed). Note that males exhibited the contrasted stripes ornament (Fig. 2, lateral display), typically described for females or for males interacting with females (Berglund \& Rosenqvist 2003).
Table 2

Multivariate tests of significance from a MANOVA of male and female interactions (approach, parallel swimming, flickering and lateral display)

\begin{tabular}{|c|c|c|c|c|c|}
\hline Effect & Wilk's $\lambda$ & $F$ & Effect $d f$ & Error $d f$ & $P$ \\
\hline \multicolumn{6}{|l|}{ Approach } \\
\hline Sex ratio & 0.60 & 15482.00 & 4 & 214 & $<0.001$ \\
\hline Sex & 1.00 & 0.24 & 2 & 107 & 0.79 \\
\hline Size & 0.50 & 53135.00 & 2 & 107 & $<0.001$ \\
\hline Sex ratio*Sex & 0.39 & 31848.00 & 4 & 214 & $<0.001$ \\
\hline Sex ratio*Size & 0.83 & 5137.00 & 4 & 214 & 0.001 \\
\hline Sex*Size & 0.98 & 1072.00 & 2 & 107 & 0.346 \\
\hline Sex ratio*Sex*Size & 0.88 & 3571.00 & 4 & 214 & 0.008 \\
\hline \multicolumn{6}{|l|}{ Parallel swimming } \\
\hline Sex ratio & 0.98 & 0.53 & 4 & 214 & 0.712 \\
\hline Sex & 0.20 & 210605.00 & 2 & 107 & $<0.001$ \\
\hline Size & 0.60 & 36265.00 & 2 & 107 & $<0.001$ \\
\hline Sex ratio*Sex & 0.03 & 249536.00 & 4 & 214 & $<0.001$ \\
\hline Sex ratio*Size & 0.92 & 2187.00 & 4 & 214 & 0.072 \\
\hline Sex*Size & 0.84 & 10060.00 & 2 & 107 & $<0.001$ \\
\hline Sex ratio*Sex*Size & 0.70 & 10276.00 & 4 & 214 & $<0.001$ \\
\hline \multicolumn{6}{|l|}{ Flickering } \\
\hline Sex ratio & 0.70 & 10321.00 & 4 & 214 & $<0.001$ \\
\hline Sex & 0.77 & 15904.00 & 2 & 107 & $<0.001$ \\
\hline Size & 0.64 & 30152.00 & 2 & 107 & $<0.001$ \\
\hline Sex ratio*Sex & 0.22 & 60784.00 & 4 & 214 & $<0.001$ \\
\hline Sex ratio*Size & 0.91 & 2705.00 & 4 & 214 & 0.031 \\
\hline Sex*Size & 0.86 & 8812.00 & 2 & 107 & $<0.001$ \\
\hline Sex ratio*Sex*Size & 0.67 & 11892.00 & 4 & 214 & $<0.001$ \\
\hline \multicolumn{6}{|l|}{ Lateral display } \\
\hline Sex ratio & 0.72 & 9592.00 & 4 & 214 & $<0.001$ \\
\hline Sex & 0.28 & 139249.00 & 2 & 107 & $<0.001$ \\
\hline Size & 0.38 & 87869.00 & 2 & 107 & $<0.001$ \\
\hline Sex ratio*Sex & 0.44 & 27465.00 & 4 & 214 & $<0.001$ \\
\hline Sex ratio*Size & 0.77 & 7490.00 & 4 & 214 & $<0.001$ \\
\hline Sex*Size & 0.52 & 50129.00 & 2 & 107 & $<0.001$ \\
\hline Sex ratio*Sex*Size & 0.47 & 24835.00 & 4 & 214 & $<0.001$ \\
\hline
\end{tabular}

Dependent variables included intra- and intersexual interactions. Analyses were performed independently for each behaviour. 
Table 3

Univariate results for each dependent variable in the MANOVA on male and female interactions (approach, parallel swimming, flickering and lateral display)

\begin{tabular}{|c|c|c|c|c|c|c|c|}
\hline \multirow[t]{2}{*}{ Effect } & & \multicolumn{3}{|c|}{ Intrasexual interactions } & \multicolumn{3}{|c|}{ Intersexual interactions } \\
\hline & & Mean square & $F$ & $P$ & Mean square & $F$ & $P$ \\
\hline \multicolumn{8}{|l|}{ Approach } \\
\hline Sex ratio & 2 & 229374 & 29.047 & $<0.001$ & 37750.509 & 7.407 & 0.001 \\
\hline Sex & 1 & 3540 & 0.448 & 0.505 & 203.276 & 0.04 & 0.842 \\
\hline Size & 1 & 519459 & 65.782 & $<0.001$ & 237515.259 & 46.604 & $<0.001$ \\
\hline Sex ratio*Sex & 2 & 427239 & 54.103 & $<0.001$ & 147953.520 & 29.031 & $<0.001$ \\
\hline Sex ratio*Size & 2 & 58402 & 7.396 & 0.001 & 16262.294 & 3.191 & 0.045 \\
\hline Sex*Size & 1 & 885 & 0.112 & 0.738 & 10192.594 & 2.000 & 0.16 \\
\hline Sex ratio*Sex*Size & 2 & 52321 & 6.626 & 0.002 & 3116.543 & 0.612 & 0.544 \\
\hline Error & 108 & 7897 & & & 5096.425 & & \\
\hline \multicolumn{8}{|l|}{ Parallel swimming } \\
\hline Sex ratio & 2 & 0.047 & 0.274 & 0.761 & 232.173 & 0.813 & 0.446 \\
\hline Sex & 1 & 711.517 & 414.989 & $<0.001$ & 676.534 & 2.370 & 0.127 \\
\hline Size & 1 & 67.415 & 39.319 & $<0.001$ & 11352.001 & 39.772 & $<0.001$ \\
\hline Sex ratio*Sex & 2 & 2838.247 & 1655.393 & $<0.001$ & 600.367 & 2.103 & 0.127 \\
\hline Sex ratio*Size & 2 & 0.592 & 3.451 & 0.035 & 360.676 & 1.264 & 0.287 \\
\hline Sex*Size & 1 & 25.290 & 14.751 & $<0.001$ & 1189.401 & 4.167 & 0.044 \\
\hline Sex ratio*Sex*Size & 2 & 26.988 & 15.741 & $<0.001$ & 1466.973 & 5.140 & 0.007 \\
\hline Error & 108 & 0.172 & & & 285.425 & & \\
\hline \multicolumn{8}{|l|}{ Flickering } \\
\hline Sex ratio & 2 & 0.918 & 1.636 & 0.2 & 35.247 & 21.182 & $<0.001$ \\
\hline Sex & 1 & 178.432 & 31.813 & $<0.001$ & 0.402 & 0.241 & 0.624 \\
\hline Size & 1 & 256.783 & 45.782 & $<0.001$ & 24.360 & 14.639 & $<0.001$ \\
\hline Sex ratio*Sex & 2 & 947.931 & 169.009 & $<0.001$ & 18.672 & 11.221 & $<0.001$ \\
\hline Sex ratio*Size & 2 & 26.390 & 4.705 & 0.011 & 1.407 & 0.846 & 0.432 \\
\hline Sex*Size & 1 & 80.753 & 14.398 & $<0.001$ & 5.447 & 3.274 & 0.073 \\
\hline Sex ratio*Sex*Size & 2 & 115.649 & 20.619 & $<0.001$ & 7.924 & 4.762 & 0.01 \\
\hline Error & 108 & 0.561 & & & 1.664 & & \\
\hline \multicolumn{8}{|l|}{ Lateral display } \\
\hline Sex ratio & 2 & 311219 & 18.814 & $<0.001$ & 9.717 & 1.873 & 0.159 \\
\hline Sex & 1 & 2136454 & 129.152 & $<0.001$ & 722.971 & 139.369 & $<0.001$ \\
\hline Size & 1 & 1701775 & 102.875 & $<0.001$ & 346.151 & 66.728 & $<0.001$ \\
\hline Sex ratio*Sex & 2 & 437911 & 26.472 & $<0.001$ & 197.914 & 38.152 & $<0.001$ \\
\hline Sex ratio*Size & 2 & 242388 & 14.653 & $<0.001$ & 5.850 & 1.128 & 0.328 \\
\hline Sex*Size & 1 & 1554312 & 93.961 & $<0.001$ & 26.328 & 5.075 & 0.026 \\
\hline Sex ratio*Sex*Size & 2 & 289986 & 17.530 & $<0.001$ & 200.789 & 38.706 & $<0.001$ \\
\hline Error & 108 & 16542 & & & 5.187 & & \\
\hline
\end{tabular}

Analyses were performed independently for each behaviour.

Within females, the excess of males resulted in a decrease in intrasexual interactions compared to the equal treatment. No female was observed swimming in parallel or flickering towards other females. Additionally, small females never displayed the ornament. Approach and lateral display were significantly affected by size, with large females being more active. An excess of females did not intensify female-female interactions. Large females displayed less than in the even treatment (significant differences for approach, parallel swimming and lateral display) while small females showed no decrease in activity and even flickered more often. Still, large females remained more active than small ones (except in approach). Within males, interactions were reduced to approach as in the equal treatment, and no differences in the level of activity were found between large and small individuals.

\section{Intersexual interactions}

The extremely low level of parallel-swimming interactions (Fig. 2) displayed by both sexes and sizes indicates that this behaviour is primarily expressed towards the same sex.

In the equal sex ratio treatment, and in contrast to intrasexual interactions which were almost exclusive to females, both sexes displayed all four behaviours towards the opposite sex with the exception of lateral display in males. Larger females performed more lateral displays and flickered more often than smaller ones.

The male-biased sex ratio had distinct effects on the way different-sized males interacted with females (Fig. 2), with only large males increasing the frequency of approach and lateral display, compared to the equal sex ratio. Within females, the more pronounced change was the absence of flickering and the reduction of lateral display. Female size only affected the frequency of approaches, with large females approaching males more often than small ones.

The female-biased sex ratio had a more subtle effect on intersexual interactions leading only to a decrease in approach by all males (small males approached females less often than large ones) and flickering by large males only. Within females, no differences were observed compared to the even sex ratio. A significant effect of size was observed on the frequency of flickering and ornament display.

\section{ACTUS and Chi-square Analysis}

\section{Courtship disruptions}

The ACTUS analysis on successful pair disruptions by males did not differ significantly from what would be expected if there was no dependence among the variables male size and sex ratio treatment $\left(\chi_{5}^{2}=1.124, P=0.576\right.$; Fig. 3$)$. Nevertheless, under a male-biased sex ratio, large males were more successful at disrupting courting pairs $\left(\chi_{1}^{2}=42.361, P<0.001\right)$. Female disruptions, on the other hand, highlighted a strong association between body size and sex ratio treatment (ACTUS; $\chi_{5}^{2}=19.905, P<0.001$; Fig. 3). Significant deviations from the expected frequencies were observed in the equal and 
Intrasexual interactions
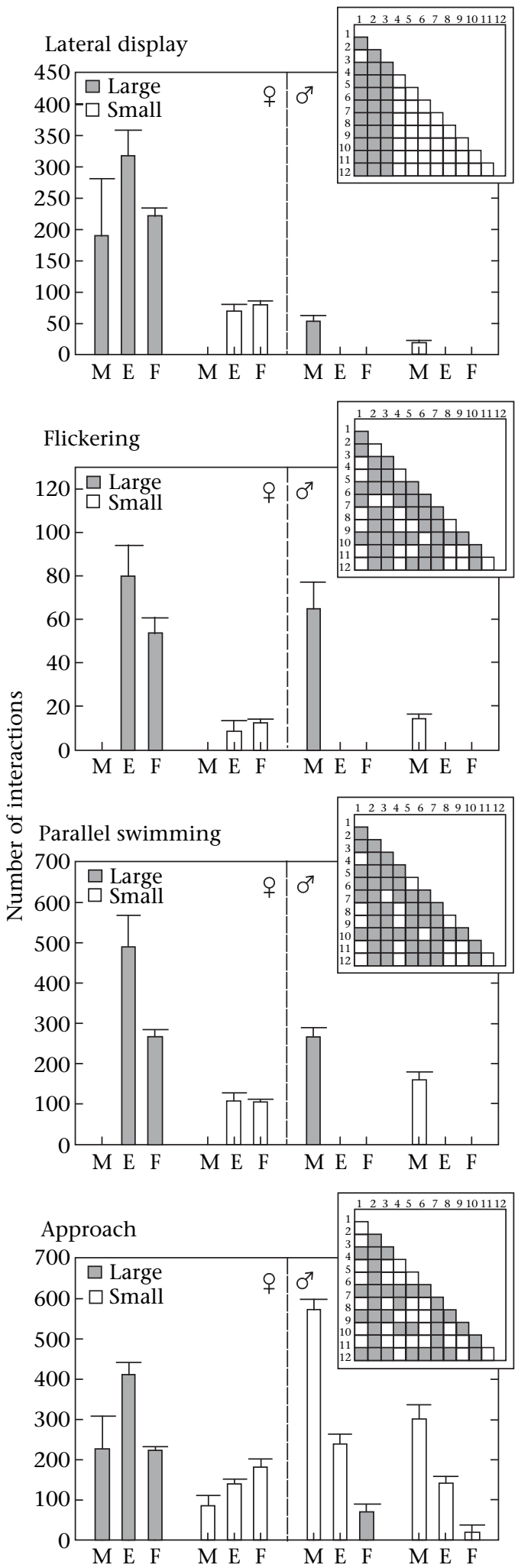

Intersexual interactions
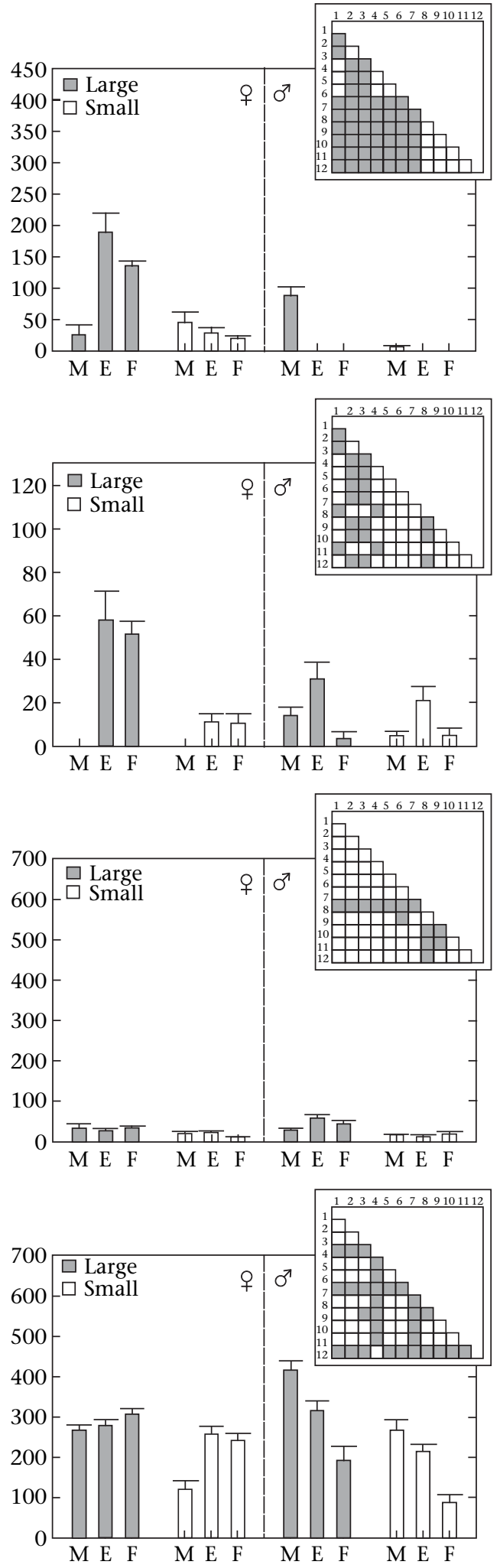

Treatment

Figure 2. Mean frequencies of all four selected behaviours for male and female inter- and intrasexual interactions in each sex ratio treatment $(E=e$ equal, $M=$ male biased and $\mathrm{F}=$ female biased). Newman-Keuls test results are presented with grey squares indicating significant differences. Error bars represent SDs. 


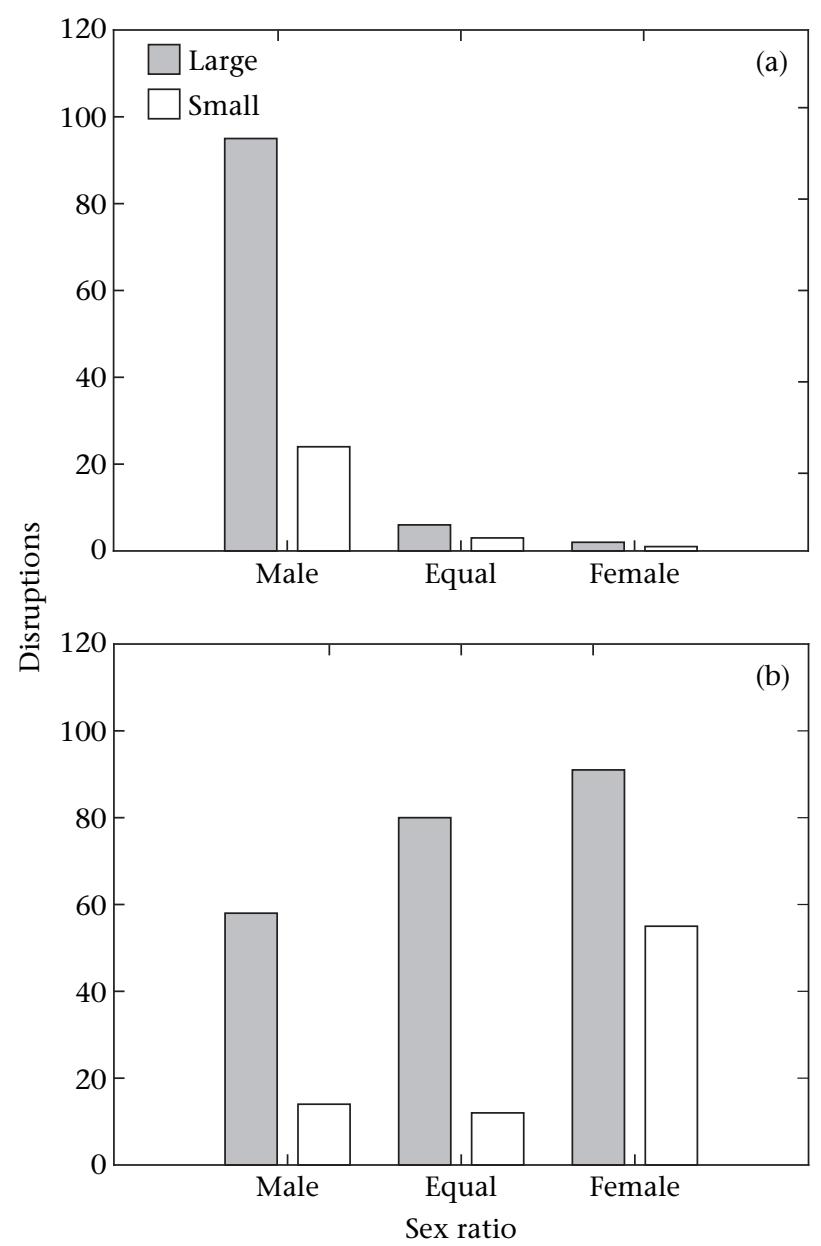

Figure 3. Number of (a) male and (b) female successful mating disruptions observed in the three selected treatments (equal, male- and female-biased sex ratios).

female-biased sex ratio treatments. More disruptions than those expected by chance were observed in the female-biased sex ratio for small females. In contrast, fewer disruptions than those expected by chance were observed in the equal sex ratio treatment for small females as well as in the female-biased sex ratio for large females.

In all treatments, large females were more successful at disrupting courting pairs (even sex ratio treatment: $\chi_{1}^{2}=50.261$, $P<0.001$; female-biased sex ratio treatment: $\chi_{1}^{2}=8.877, P<0.01$; male-biased sex ratio treatment: $\chi_{1}^{2}=26.889, P<0.001$ ).

\section{Mating pairs}

Size-matched pairs were always more frequent than pairs formed by individuals of contrasting size classes (Fig. 4), independently of sex ratio treatment (ACTUS: $\chi_{5}^{2}=1.551, P=0.481$; equal sex ratio: 33/10 pairs; female-biased sex ratio: $16 / 4$ pairs; malebiased sex ratio: $17 / 9$ pairs). For the less common nonsize-matched pairs, a significant association was observed between sex ratio treatment and the relative size combination of males and females (ACTUS: $\chi_{5}^{2}=13.081, P<0.001$ ). In the female-biased sex ratio, where males were scarce and females more abundant, large males did not mate with small females. Similarly, in the male-biased sex ratio, where males were in excess, large females did not mate with small males. While small males mated more often with large females in the female-biased sex ratio, the opposite (small females mating with large males in the male-biased sex ratio) did not occur more frequently than expected by chance alone.

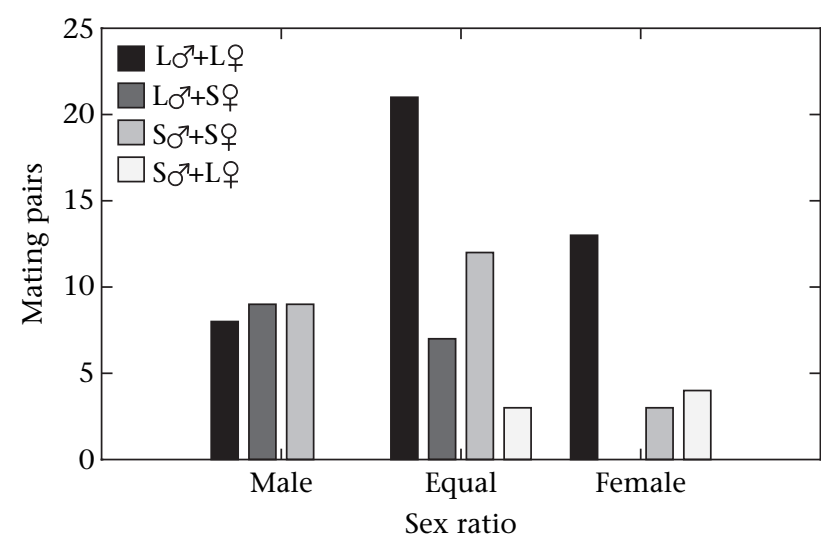

Figure 4. Number of mating pairs observed in the three selected treatments (equal, male- and female-biased sex ratios), described according to the four possible size class combinations of males and females ( $\mathrm{L}=$ large, $\mathrm{S}=$ small).

ANOVA

Finally, the ANOVA results on the time taken to the first mating, considering the sex ratio treatment and male size, revealed a significant effect of sex ratio (Table 4, Fig. 5). No significant interaction with male size was observed. The time taken until the first spawning event was significantly higher in the female-biased sex ratio than in the other treatments.

\section{DISCUSSION}

There is increasing evidence that mating patterns are, in general, more plastic than previously thought (e.g. Kvarnemo et al. 1995; Forsgren et al. 2004). The present study has provided evidence of some of the effects of sex ratio variation on the reproductive behaviour of $S$. abaster males and females. The sex role reversal described for this species (Silva et al. 2006) could be easily observed in the even sex ratio treatment, where females were clearly more competitive than males, investing intensely in intrasexual displays, probably as a means to establish a dominance hierarchy based on body size (see also Silva et al. 2007). In fact, besides engaging more in intrasexual interactions, large dominant females were also far more successful than small ones at disrupting ongoing courtships. Males, on the other hand, seemed to devote their efforts solely to courting females. As mentioned in Silva et al. (2006), this active role in courtship contrasts with other sex role-reversed pipefishes where males are much more passive (e.g. Nerophis lumbriciformis: Monteiro et al. 2002).

Even taking into consideration that $S$. abaster is a sex rolereversed species (at least under an even sex ratio), it could be expected that a surplus of males would boost male-male interactions, probably even reversing sex role reversal, since females would become the limiting sex. Our results seem to corroborate this prediction since the level of male intrasexual interactions rose significantly above that of female-female interactions, under

Table 4

ANOVA results on the mean time elapsed until the first mating event, considering sex ratio treatment and male size

\begin{tabular}{lrclc}
\hline Effect & $d f$ & Mean square & $F$ & $P$ \\
\hline Size & 1 & 0.05 & 0.04 & 0.84 \\
Sex ratio & 2 & 11.50 & 8.65 & $<0.001$ \\
Size*Sex ratio & 2 & 0.40 & 0.30 & 0.74 \\
Error & 69 & 1.33 & & \\
\hline
\end{tabular}




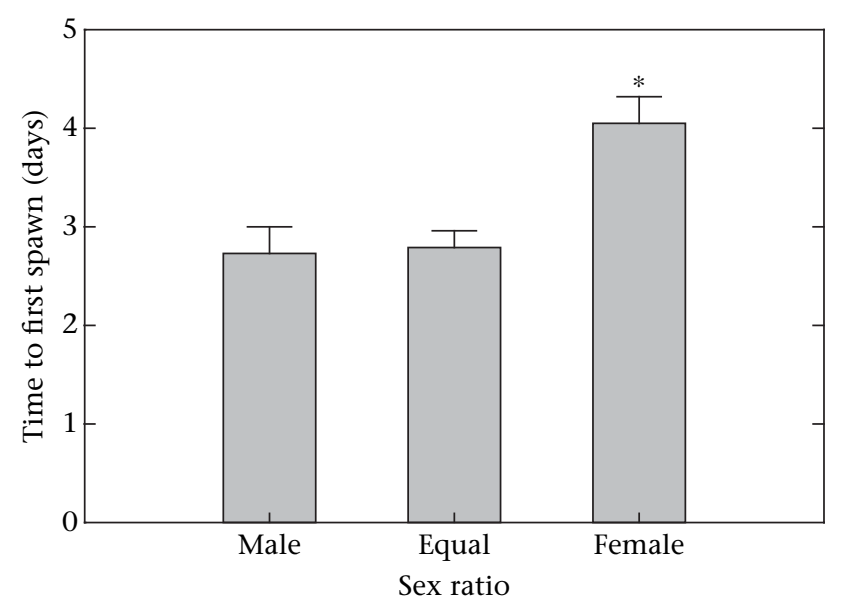

Figure 5. Mean time elapsed until the first spawn in the three selected treatments (equal, male- and female-biased sex ratios). Error bars represent SDs. ${ }^{*} P<0.05$.

a male-biased sex ratio. Males were observed displaying the ornament towards other males, a phenomenon that, at least to our knowledge, has never been described for syngnathids. Berglund \& Rosenqvist (2001) stated that the male's ornament might have evolved as a correlated response to selection for ornamentation in females, as males prefer ornamented females. Nevertheless, the male ornament has already been described as a means of signalling females in the sex role-reversed pipefish S. typhle (Berglund et al. 2005). Surprisingly, S. abaster males seem capable of using the ornament in both contexts, courtship and competition. Since ornament display was only observed under the male-biased sex ratio, the treatment where males actively competed for female access, it could be argued that ornament function in males is similar to that of female displays: signalling quality not only towards the opposite sex but also to potential rivals. As well as in females (Silva et al. 2007), male size seems also to play a major role in the outcome of intrasexual interactions, mainly in the male-biased sex ratio, where male competition occurs. Larger males interacted more than small ones (significant differences were observed in all the behaviours except lateral display) and were also more successful at disrupting mating pairs. Thus, the male dominance hierarchy seems similar to that of females, based on size and advertised with the same ornament.

Surprisingly, a shift towards a female-biased sex ratio did not globally intensify female-female competition. Contrary to what could be expected, large females, usually dominant and prone to intrasexual competitive behaviours, interacted even less with other females (with the exception of flickering where no differences were observed). Small females, on the other hand, showed no reduction in the number of interactions, and flickered more in the femalebiased treatment. Since, when many females were available, males seemed to be 'more choosy', preferring larger partners and avoiding mating with smaller ones, it could be hypothesized that large (preferred) females might not need to maintain or increase competitive behaviours, as small ones do, to obtain a mating partner since male choosiness translates into a higher reproductive confidence. In contrast, small females continued to try to obtain a mating partner (e.g. flickering rose significantly from the even to the female-biased sex ratio). The more active behaviour of small females in this treatment was also translated into an increased courtship activity. These results seem consistent with previous work on this species which highlighted a switch point in the female's reproductive tactics over her lifetime (Silva et al. 2009). Berglund (1991) showed that smaller S. typhle females seem to trade immediate reproduction for growth to increase the prospects of future reproductive success. Rather than an intrinsic speciesspecific difference in the response to demographic structure between these two species, the decision to allocate resources to reproduction or growth might also be closely linked to the extent of the breeding season experienced in the sampled populations. In Portugal, S. abaster's breeding season is almost twice as long as that experienced by the Swedish populations of $S$. typhle, thus increasing the chances of small females finding a mate even if that implies waiting for large, preferred females to stop reproducing.

Game-theoretical models predict that the value of winning should affect how much an individual invests in a contest (Leimar et al. 1991). Accordingly, Parker (1983) suggested that individuals might vary their mating tactics according to their relative attractiveness. In S. typhle, small males, which have a lower competitive ability, courted more and took greater risks to get a partner, than larger, preferred males (Billing et al. 2007). Also, in the Trinidadian guppy, Poecilia reticulata, attractive males showed lower levels of courtship as they gained higher reproductive benefits compared to less attractive individuals (Reynolds 1993). Thus, when exposed to few potential mates, large female $S$. abaster might opt to decrease competition, since temporarily postponing reproduction and waiting for additional mating opportunities might not be a high-risk strategy given male preference for large partners. In a parallel experiment, large females laid more eggs when surrounded by a large number of males (Silva et al. 2009). Thus, it seems that the number of available males is an important variable in the expression of female mating behaviour.

It could be expected that a skewed sex ratio towards one sex, besides increasing intrasexual competition, would also intensify courtship. Nevertheless, the most drastic alterations were observed in intrasexual interactions, rather than courtship, a fact especially surprising considering that the monitored behaviours are usually described as part of the courtship ritual. Similar findings were obtained in the sand goby where behavioural patterns connected to courtship did not change with the sex ratio, while competitive interactions were drastically affected (Kvarnemo 1996). One possible explanation for this observation might lie in the fact that intrasexual interactions may also serve as a cue for mate choice. Animals obtain information and guide their choice between potential partners from observing competitive interactions and displays between them, just as well as from displays directed towards the choosing individual (Kvarnemo et al. 1995; Berglund \& Rosenqvist 2001). In S. typhle, for example, males preferred dominant over attractive females, remembering information from competitive displays and using it, rather than immediate information from displays (Berglund \& Rosenqvist 2001).

Field observations revealed a size-assortative mating pattern in S. abaster (Silva et al. 2007), which was also visible in our present aquarium experiment. Independently of sex ratio treatment, individuals mated more often with similar-size partners. When we analysed only pairs formed by individuals of contrasting sizes, it became apparent that large individuals were especially choosier when there was an excess of mating partners. Small individuals, on the other hand, might not have the opportunity to be as choosy as large ones since both sexes have similar preferences for large partners (Silva et al. 2006). Small females might be especially limited since large females appear to monopolize male access. As an example, small males mated significantly more often than could be expected with large females in the female-biased sex ratio. In the male-biased sex ratio, small females did not mate with large males more often than expected.

Male choosiness was also seen in the female-biased sex ratio where time required until the first egg transfer was significantly higher. A similar observation has been made in S. typhle where male choosiness appears to be affected by the perceived proportion of 
potential mates and competitors (Berglund 1994). Berglund (1994) suggested that this allows males to modify their mating strategy to prevailing OSRs in nature.

A hypothetical delay in egg transfer could also be expected in the male-biased sex ratio, where females are choosier. Since this delay was not observed, it seems justifiable to assume that males, more than females, ultimately control the egg transfer event.

Sex ratio proved to be an effective variable in the expression of sex roles, with a surplus of males being able to disrupt the sex role reversal normally observed when both sexes coexist in similar numbers. Indeed, our results showed that male and female $S$. abaster seem able to detect imbalances in the 'population' sex ratio actively, becoming both choosier and less competitive towards conspecifics when the opposite sex is in excess, or more prone to adopt a competitive strategy. In specific scenarios where hypothetical costs derived from intrasexual competition might outweigh the benefits of immediately acquiring a partner, large females seem to count on their 'sex appeal', becoming less competitive towards conspecifics, probably saving resources for future breeding events.

Altogether, our results show a highly dynamic system, far from what could be expected under a binary sex role definition where individuals are also usually assumed to be solely competitive or choosy. Given these results, it could be argued that cases of apparently discordant sex roles, as in S. schlegeli (reversed according to Watanabe et al. 2000 under a female-biased sex ratio, and conventional according to Kornienko 2001 under an even sex ratio), might just reflect a behavioural plasticity, similar to that described for S. abaster.

\section{Acknowledgments}

We thank Gunilla Rosenqvist and two anonymous referees for their valuable comments on the manuscript. We also thank everybody that helped during the laboratory work, especially Pedro Correia. N.M.M. was funded by Fundação para a Ciência e a Tecnologia (FCT-SFRH/BPD/14992/2004). K.S. was funded by Fundação para a Ciência e a Tecnologia (FCT-SFRH/BD/13171/2003). This work was partially funded by FCT (POCI/MAR/60895/2004).

\section{References}

Almada, V. C., Gonçalves, E. J., Oliveira, R. F. \& Santos, A. J. 1995. Courting females: ecological constraints affect sex roles in a natural population of the blenniid fish Salaria pavo. Animal Behaviour, 49, 1125-1127.

Berglund, A. 1991. Egg competition in a sex-role reversed pipefish: subdominant females trade reproduction for growth. Evolution, 45, 770-774.

Berglund, A. 1994. The operational sex ratio influences choosiness in a pipefish. Behavioural Ecology, 5, 254-258.

Berglund, A. \& Rosenqvist, G. 2001. Male pipefish prefer dominant over attractive females. Behavioral Ecology, 12, 402-406.

Berglund, A. \& Rosenqvist, G. 2003. Sex-role reversal in pipefish. Advances in the Study of Behavior, 32, 131-167.

Berglund, A., Sandvik, W. M. \& Rosenqvist, G. 2005. Sex-role reversal revisited: choosy females and ornamented, competitive males in a pipefish. Behavioral Ecology, 200, 649-655.

Berglund, A., Rosenqvist, G. \& Robinson-Wolrath, A. 2006. Food or sex: males and females in a sex role reversed pipefish have different interests. Behavioral Ecology and Sociobiology, 60, 281-287.

Billing, A. M., Rosenqvist, G. \& Berglund, A. 2007. No terminal investment in pipefish males: only young males exhibit risk-prone courtship behavior. Behavioral Ecology, 18, 535-540.
Cakic, P., Lenhardt, M., Mickovic, D., Sekulic, N. \& Budakov, L. J. 2002. Biometric analysis of Syngnathus abaster populations. Journal of Fish Biology, 60, 1562-1569.

Clutton-Brock, T. H. 2007. Sexual selection in males and females. Science, 318, 1882-1885.

Clutton-Brock, T. H. \& Parker, G. A. 1992. Potential reproductive rates and the operation of sexual selection. Quaterly Review of Biology, 67, 437-456.

Dawson, C. E. 1986. Syngnathidae. In: Fishes of the North-eastern Atlantic and the Mediterranean (Ed. by P. J. P. Whitehead, M. L. Bauchot, J. C. Hureau, J. Nielsen \& E. Tortonese), pp. 628-639. Paris: UNESCO.

Emlen, S. T. \& Oring, L. W. 1977. Ecology, sexual selection, and the evolution of mating systems. Science, 197, 215-223.

Enders, M. M. 1993. The effect of male size and operational sex ratio on male mating success in the common spider mite, Tetranychus urticae Kock (Acari: Tetranychidae). Animal Behaviour, 46, 835-846.

Estabrook, C. B. \& Estabrook, G. F. 1989. ACTUS: a solution to the problem of small samples in the analysis of two-way contingency tables. Historical Methods, 22, 5-8.

Forsgren, E., Amundsen, T., Borg, A. A. \& Bjelvenmark, J. 2004. Unusually dynamic sex-roles in a fish. Nature, 429, 551-554.

Grant, J. W. A. 1995. Operational sex ratio, mediated by synchrony of female arrival, alters the variance of male mating success in Japanese medaka. Animal Behaviour, 49, 367-375.

Gwynne, D. T. \& Simmons, L. W. 1990. Experimental reversal of courtship roles in an insect. Nature, 346, 172-174.

Jones, A. G., Rosenqvist, G., Berglund, A. \& Avise, J. C. 2000. Mate quality influences multiple maternity in the sex-role-reversed pipefish Syngnathus typhle. Oikos, 90, 321-326.

Jones, A. G., Rosenqvist, G., Berglund, A. \& Avise, J. C. 2005. The measurement of sexual selection using Bateman's principles: an experimental test in the sexrole-reversed pipefish Syngnathus typhle. Integrative and Comparative Biology, 45, 874-888.

Kokko, H. \& Monaghan, P. 2001. Predicting the direction of sexual selection. Ecology Letters, 4, 159-165.

Kornienko, E. S. 2001. The spawning behaviour of the pipefish Syngnathus acusimilis. Russian Journal of Marine Biology, 27, 54-57.

Kvarnemo, C. 1996. Temperature affects operational sex ratio and intensity of malemale competition: an experimental study on sand gobies. Behavioral Ecology, 7, 208-212.

Kvarnemo, C., Forsgren, E. \& Magnhagen, C. 1995. Effects of sex ratio on intra- and intersexual behaviour in sand gobies. Animal Behaviour, 50, 1455-1461.

Leimar, O., Austad, S. \& Enquist, M. 1991. A test of the sequential assessment game: fighting in the bowl and doily spider Frontinella pyramitela. Evolution, 45, 862-874.

Lindman, H. R. 1974. Analysis of Variance in Complex Experimental Designs. San Francisco: W.H. Freeman.

Monteiro, N. M., Vieira, N. M. \& Almada, V. C. 2002. The courtship behaviour of the pipefish Nerophis lumbriciformis: reflections of an adaptation to intertidal life. Acta Ethologica, 4, 109-111.

Parker, G. A. 1983. Mate quality and mating decisions. In: Mate Choice (Ed. by P. Bateson), pp. 141-166. Cambridge: Cambridge University Press.

Reynolds, J. D. 1993. Should attractive individuals court more? Theory and a test American Naturalist, 141, 914-927.

Silva, K., Monteiro, N. M., Vieira, M. N. \& Almada, V. C. 2006. Reproductive behaviour of the black-striped pipefish, Syngnathus abaster (Pisces; Syngnathidae). Journal of Fish Biology, 69, 1860-1869.

Silva, K., Vieira, M. N., Almada, V. C. \& Monteiro, N. M. 2007. The effect of temperature on mate preferences and female-female interactions in Syngnathus abaster. Animal Behaviour, 74, 1525-1533.

Silva, K., Almada, V. C., Vieira, M. N. \& Monteiro, N. M. 2009. Female reproductive tactics in a sex-role reversed pipefish: scanning for male quality and number. Behavioural Ecology, 20, 768-772.

Souroukis, K. \& Cade, W. H. 1993. Reproductive competition and selection on male traits at varying sex ratios in the field cricket, Gryllus pennsylvanicus. Behaviour, 126, 45-62.

Vincent, A. C. J., Ahnesjo, I., Berglund, A. \& Rosenqvist, G. 1992. Pipefishes and seahorses: are they all sex-role reversed? Trends in Ecology E Evolution, 7, 237-241.

Vincent, A. C. J., Ahnesjo, I. \& Berglund, A. 1994. Operational sex ratios and behavioural sex differences in a pipefish population. Behavioral Ecology and Sociobiology, 34, 435-442.

Watanabe, S., Hara, M. \& Watanabe, Y. 2000. Male internal fertilization and introsperm-like sperm of the seaweed pipefish (Syngnathus schlegeli). Zoological Science, 17, 759-767. 